\title{
CT-PET Landmark-based Lung Registration Using a Dynamic Breathing Model
}

\author{
S. Chambon ${ }^{1}$, A. Moreno ${ }^{1}$, A. P. Santhanam ${ }^{2,3}$, J. P. Rolland ${ }^{2}$, E. Angelini ${ }^{1}$, I. Bloch ${ }^{1}$ \\ ${ }^{1}$ ENST, GET - Télécom Paris, CNRS UMR 5141 LTCI - Paris, France \\ ${ }^{2}$ ODALab, University of Central Florida,USA \\ ${ }^{3}$ Department of Radiation Oncology, MD Anderson Cancer Center Orlando, USA \\ \{Sylvie.Chambon,Antonio.Moreno,Elsa.Angelini,Isabelle.Bloch\}@enst.fr , \{anand,jannick\}@ odalab.ucf.edu
}

\begin{abstract}
This paper deals with the problem of non-linear landmark-based registration of CT (at two different instants of the breathing cycle, intermediate expirations) and PET images of thoracic regions. We propose a general method to introduce a breathing model in a registration procedure in order to simulate the instant in the breathing cycle most similar to the PET image and guarantee physiologically plausible deformations. Initial results are very promising and demonstrate the interest of this method to improve the combination of anatomical and functional images for diagnosis and oncology applications.
\end{abstract}

\section{Introduction}

Registration of multimodal medical images is a widely addressed topic and is important in many different domains, in particular for oncology and radiotherapy applications. We consider Computed Tomography (CT) and Positron Emission Tomography (PET) of thoracic regions, which provide complementary information about the anatomy and the metabolism of the human body (see Figure 1). Their registration has a significant impact on improving medical decisions for diagnosis and therapy [7, 14, 23]. Linear registration is not sufficient to cope with the local deformations produced by cardiac and respiratory motions. Therefore, non-linear registration methods are required to register multimodality images of thoracic and abdominal regions, even with combined PET/CT scanners [21].

Most of the existing non-linear registration methods use intensity information or features in order to calculate the transformation between the images $[8,13,26]$. Thus they have to either find the transformation that maximizes the similarity between the registered image and the target image (iconic methods) or compute a transformation that matches some particular features (landmarks) in both images (geometrical methods). In the case of landmark-based methods, the selection of these particular features is an important task. In many methods, the curvature of the surfaces to reg- ister is used. However, few papers included a detailed study of the selection of the landmarks [3, 16]. Moreover, most registration methods are based on image information, and do not take into account the physiology of the human body. However, physiological information can be useful in order to ensure realistic deformations and to guide the registration process. While several papers present breathing models built for medical visualization [27], no paper exploits such a model in a registration process. Consequently, in this paper, we propose an approach in which we integrate a physiologically driven breathing model in a non-linear registration procedure based on lung surface landmark points in order to guarantee physiologically plausible deformations.

In Section 2, we summarize existing works which use breathing models combined (or not) with registration algorithms and then we provide an overview of the selected model. The proposed model-based non-linear registration algorithm is detailed in Section 3. Then, the application of a landmark-based registration method adapted to pathological cases combined with the breathing model is described in Section 4. Section 5 discusses some results.

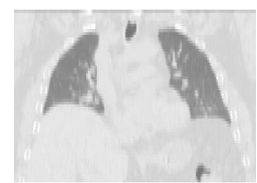

(a)

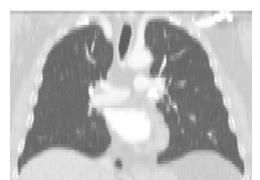

(b)

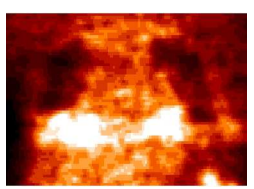

(c)
Figure 1. Corresponding views of the same patient in CT (a)-(b) for two instants of the breathing cycle, and PET (c).

\section{Breathing Models}

\subsection{Thoracic Imaging Registration}

A recent study highlighted the effects of breathing during a non-rigid registration process and the importance of taking these into account [22]. Three techniques for respiration-gated radiotherapy are being developed to improve the efficiency of lung tumors radiations:

(1) active: controling the patient's breathing via airflow blockage; 
(2) passive or empirical: using external measurements in order to adapt radiation protocols to the tumor's motion $[9,12,25]$;

(3) model-based: employing a breathing model to predict lungs deformations during the breathing cycle [19].

We focus on thoracic volume registration and propose a "patient-specific" registration through the use of a breathing model (technique (3)). Different bio-mathematical representations of the human respiratory mechanics have been developed [10]. Mathematical tools can be employed and the most popular technique, for medical visualization, is called NCAT (NURBS-based cardiac-torso). It is based on Non-Uniform Rational B-Spline (NURBS) to correct for respiratory artifacts of SPECT images [20]. A multiresolution registration for 4D Magnetic Resonance Imaging (MRI) was proposed in [15]. In [5], a 4D NCAT phantom and a 3D CT image were used to generate 4D CT and to compute an elastic registration. Physically-based models describe the important role of airflow inside the lungs and can be based on Active Breathing Coordinator (ABC) [19] or volume preservation relation [17, 27]. Authors of $[15,22]$ used pre-register MRI to estimate the breathing model. In [5], the NCAT phantom was used, but, from a modeling and simulation point of view, physicallybased deformation methods are better adapted for simulating lung dynamics as they allow precise generation of intermediate 3D lung shapes. They are easy to adapt to patients, without the need for physical external adaptations for each radiotherapy treatment.

\subsection{Physics-Based Breathing Model}

The modeling approach used in this work was previously discussed in [17] and the two major components involved in the modeling and visualization efforts include:

(1) the parameterization of Pressure-Volume (PV) data of a human subject which act as an $\mathrm{ABC}$;

(2) the estimation of the deformation operator from either 4D (3D+t) CT lung data or two 3D CT lung data set.

In step (1) a parameterized PV curve, obtained from normal human subjects, is used as a driver for simulating the 3D lung shapes at different lung volumes. In step (2), the computation takes as inputs the nodal displacements of the 3D lung model and the estimated amount of force applied on the nodes of the meshes (which are on the surface). Displacements are obtained from 4D CT of a normal human subject. The direction and magnitude of the lung surface point's displacement are computed using the fact that the expansion of lung tissues is linearly related to the increase in lung volume. The estimated amount of applied force on each node (that represents the air-flow inside lungs) is estimated based on a PV curve and the lungs's orientation with respect to the gravity, which controls the air flow. Given these inputs, a physics-based deformation approach based on Green's function (GF) formulation is estimated to deform the 3D lung surface models. Specifically the GF is defined in terms of a physiological factor, the regional alveolar expandability (elastic properties), and a structural factor, the inter-nodal distance of the 3D surface lung model. To compute the coefficients of these two factors, an iterative approach is employed and, at each step, the force applied on a node is shared with its neighboring nodes based on a local normalization of the alveolar expandability coupled with inter-nodal distance. The process stops when sharing of the applied force reaches equilibrium [18].

\section{Using the Breathing Model in Registration}

We have conceived an original algorithm in order to introduce the advantages of using the breathing model described above in a registration procedure. Figure 2 shows the computational workflow of the complete algorithm. The inputs consist of one PET volume and two CT volumes of the same patient, corresponding to two different instants of the breathing cycle (intermediate expirations). The first step of the algorithm consists in segmenting the lungs and the tumors on the PET data and on the two CT data sets, using a robust mathematical-morphology-based approach [4]. The meshes (called CT mesh and PET mesh) corresponding to the different segmentation results are computed. The subsequent steps are detailed next.

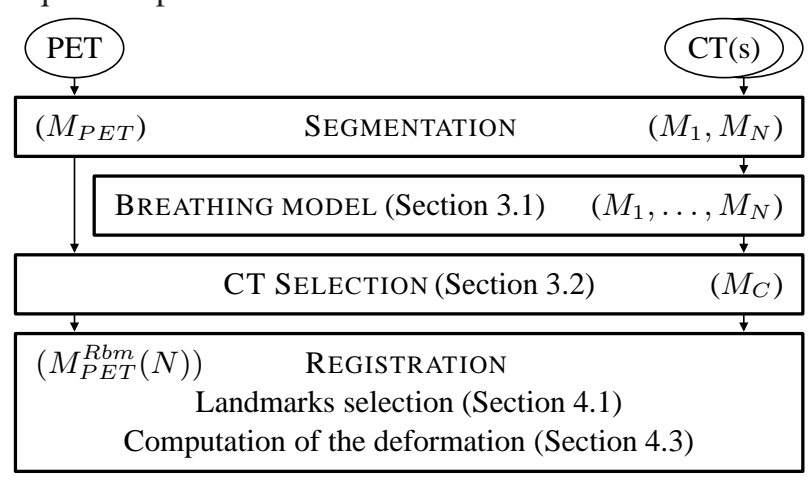

Figure 2. Computational workflow of the registration of CT and PET images using a breathing model (cf. Section 3.3).

\subsection{Patient-Specific Breathing Model}

We first estimate the intermediate 3D lung shapes between the two segmented CT lung datasets. Displacements of lung surface points are computed as follows:

(1) Directions are given by the model (computed from a 4D CT normal data set of reference).

(2) Magnitudes are "patient-specific" and are computed from the given 3D CT lung datasets.

In other words, for known directions of displacement the magnitude of the displacement is computed from the two 3D CT lung datasets. With known estimations of applied 
force and "subject-specific" displacements the coefficients of the GF can be estimated (cf. Section 2.2). Then, the GF operator is used to compute the $3 \mathrm{D}$ lung shapes at different intermediate lung volumes. This methodology is further detailed in [17].

\subsection{CT Selection}

By applying the continuous breathing model, we can obtain different instants ("snapshots") of the breathing cycle, generating simulated CT meshes. By comparing each CT mesh with the PET mesh, we select the "closest" one. Let us denote the CT simulated meshes by $M_{1}, M_{2}, \ldots$, $M_{N}$. The mesh $M_{N}$ corresponds to the CT in maximum inhalation and $M_{1}$ to maximum exhalation. By using the breathing model, the transformation $\phi_{i j}$ between any two instants $i$ and $j$ of the breathing cycle can be computed as: $M_{j}=\phi_{i j}\left(M_{i}\right)$. We can compare these CT meshes with the PET mesh $\left(M_{P E T}\right)$. We define a measure of similarity between meshes (or their corresponding volumes) and the mesh that minimizes the criterion $(C)$ is denoted as $M_{C}$ :

$$
M_{C}=\arg \min _{i} C\left(M_{i}, M_{P E T}\right) .
$$

The Root Mean Square (RMS) distance has been chosen as the criterion $C$, as a first approach: $D_{R M S}(M, A)=\sqrt{\frac{1}{2}\left[d_{R M S}(M, A)^{2}+d_{R M S}(A, M)^{2}\right]}$ with $d_{R M S}(M, A)=\sqrt{\frac{1}{|M|} \sum_{p \in M} D(p, A)^{2}}$ and where $D(p, A)=\left[\min _{q \in A} d(p, q)\right]$ with $d$ the Euclidean distance.

\subsection{Deformation of the PET}

Registration of $M_{P E T}$ and the original CT mesh $M_{N}$ can be perform with two approaches :

(1) A direct registration (dashed line in Figure 3):

$$
M_{P E T}^{R d}(N)=f^{R d}\left(M_{P E T}, M_{N}\right),
$$

where $f^{R d}$ denotes the transformation that registers directly $M_{P E T}$ and $M_{N}$, and $M_{P E T}^{R d}$ the result of registering the PET directly to the CT mesh $M_{N}$. The transformation $f^{R d}$ may be computed by any registration method adapted to the problem. As an illustrative example, we choose the original CT to correspond to the end-inspiration, $M_{N}$, but a similar process could be applied for any CT image. In this approach the deformation itself is not guided by any anatomical knowledge. In addition, if the PET and the original CT are very different (end-inspiration CT), it is likely that this registration will provide physically unrealistic results.

(2) To avoid such potential problems, we propose here an alternative approach: once the appropriate CT $\left(M_{C}\right)$ is selected, we compute the registration between the $M_{P E T}$ mesh and the $M_{C}$ mesh as:

$$
M_{P E T}^{r}(C)=f^{r}\left(M_{P E T}, M_{C}\right),
$$

where $f^{r}$ is the registration transformation and $M_{P E T}^{r}$ denotes the registered mesh. Then, the transformation due to the breathing is used to register the $M_{P E T}^{r}$ to the original CT (continuous line in Figure 3). The transformation due to the breathing between $M_{C}$ and $M_{N}$ can be computed as the following composition:

$$
\Phi_{C, N}=\phi_{N-1, N} \circ \ldots \circ \phi_{C+1, C+2} \circ \phi_{C, C+1} .
$$

We apply to $M_{P E T}^{r}$ the same transformation $\Phi_{C, N}$ in order to compute the registration with $M_{N}$ :

$$
\begin{aligned}
M_{P E T}^{R b m}(N) & =\Phi_{C, N}\left(M_{P E T}^{r}(C)\right) \\
& =\Phi_{C, N}\left(f^{r}\left(M_{P E T}, M_{C}\right)\right),
\end{aligned}
$$

where $M_{P E T}^{R b m}$ denotes the PET registered mesh using the breathing model.

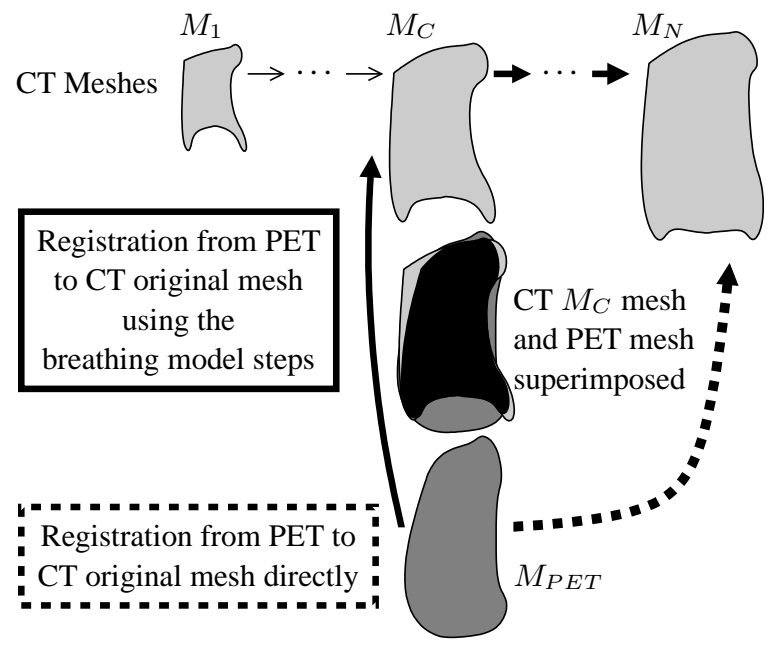

Figure 3. The mesh $M_{C}$ is the closest to the mesh $M_{P E T}$. We can register $M_{P E T}$ to $M_{N}$ following one of the two paths (notations are defined in Section 3.3).

\section{Registration Adapted to Pathologies}

The algorithm described in Section 3 can be applied with any type of registration method. We show how the proposed approach can be applied for landmark-based registration of multimodality images in pathological cases, in particular for diagnosis, follow-up and radiotherapy treatments.

\subsection{Influence of Selected Landmark Points}

Features selection is an important task in registration. In this section, we focus on voxel selection but more complex features can be detected [1]. The selection can be manual (as in most methods) [24], semi-automated [16], or automated [16]. Manual selection is tedious and timeconsuming. The authors in [6] suggest that semi-automated selection is interesting, integrating experts knowledge in an automatic process. Automatic selection permits reduced execution time with high accuracy. Most of these automatic 


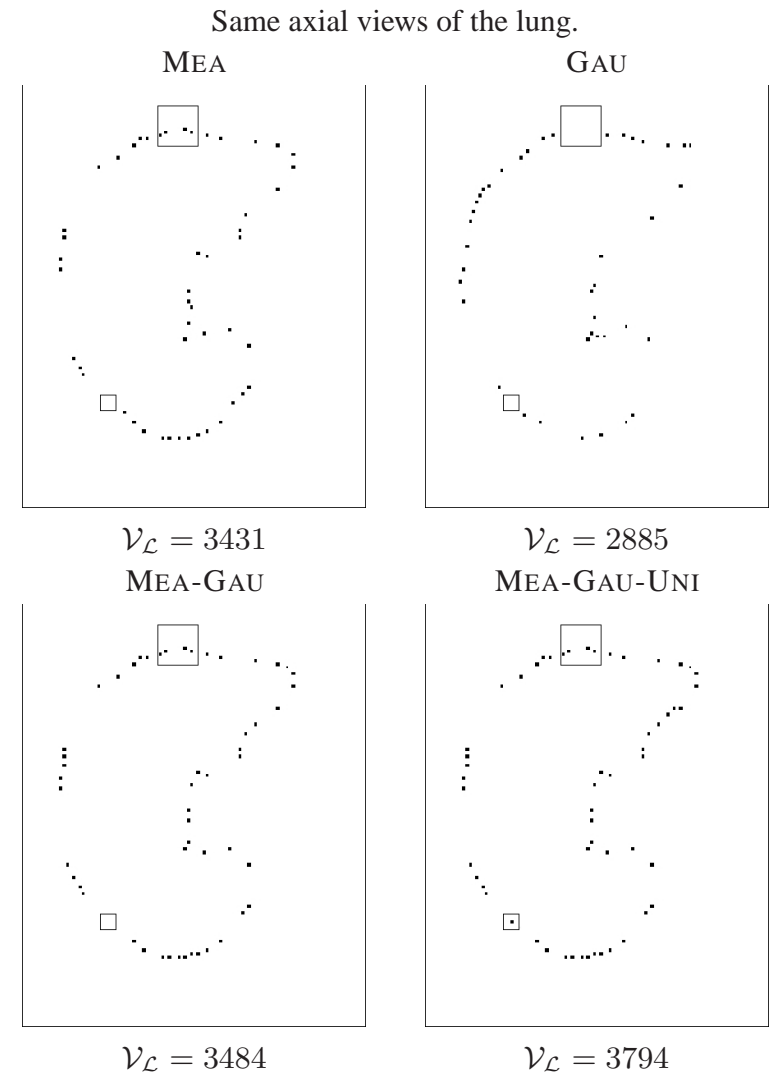

Figure 4. Selection of landmarks - In each image, two regions of interest are identified with two rectangles. In the large rectangle, there is no landmark with GAU method whereas there are four landmarks with the MEA method. In the fusion method (MEA-GAU), these landmarks are selected. In the small rectangle, no landmark is selected with the mean and/or the Gaussian curvatures. However, a landmark is added in this area with the MEA-GAU-UNI method.

methods exploit curvature [16]. In [3], an auto-correlation method is also combined with curvature.

In the present work, landmark selection is automatic and based on Gaussian and mean curvatures, according to the following steps:

(1) compute curvature for each voxel of the lung surface;

(2) sort voxels in decreasing order of absolute value of curvature;

(3) select voxels based on curvature and distance criteria (detailed in the following paragraph);

(4) if a uniform selection is needed then add voxels with zero-curvature in the area where no voxels have been considered as landmarks.

This algorithm is proposed to select particular voxels that provide relevant information. Moreover, we intend to obtain an approximately uniform selection to take into account the entire surface of the lungs for computing the deformation. In step 3 , we consider $\mathcal{V}=\left\{\mathbf{v}_{i}\right\}_{i=0 . . N_{\mathcal{S}}}$, the set of voxels in decreasing order of absolute value of curvature, where $N_{\mathcal{S}}$ is the number of voxels of the surface and $\mathcal{V}_{\mathcal{L}}=\left\{\mathbf{v}_{\mathcal{L}}\right\}_{i=0 . . N_{\mathcal{L}}}$, the set of landmarks, where $N_{\mathcal{L}}$ is the number of landmarks. For each voxel $\mathbf{v}_{i} \in \mathcal{V}$ (for $i=0$ to $N_{\mathcal{S}}$ ) with non-zero-curvature, we add $\mathbf{v}_{i}$ in $\mathcal{V}_{\mathcal{L}}$, if $\forall \mathbf{v}_{j} \in \mathcal{V}_{\mathcal{L}}, d_{g}\left(\mathbf{v}_{i}, \mathbf{v}_{j}\right)>T$ where $d_{g}$ is the geodesic distance on the lung surface and $T$ is a threshold to be chosen. With this selection process, some regions (the flattest) may contain no landmark, hence the addition of step 4: for each voxel on the surface of the lung $\mathbf{v}_{i} \in \mathcal{V}$ with zero-curvature, if there is no voxel $\mathbf{v}_{j} \in \mathcal{V}_{\mathcal{L}}$ with $d_{g}\left(\mathbf{v}_{i}, \mathbf{v}_{j}\right)<T$, we add $\mathbf{v}_{i}$ in $\mathcal{V}_{\mathcal{L}}$.

Four variants are tested:

(1) MEA - Mean curvature without step 4;

(2) GAU - Gaussian curvature without step 4;

(3) MEA-GAU - Using mean and Gaussian curvature without step 4;

(4) MeA-GAU-UnI - Using mean and Gaussian curvature with step 4.

When mean and Gaussian curvatures are employed (methods MeA-GaU and MeA-GAU-UnI), the set $\mathcal{V}$ merges the set of voxels in decreasing order of mean curvature and the set of voxels in decreasing order of Gaussian curvature, by taking alternatively a value in each set. These strategies for landmark point selection are compared in Figure 4. Results given by the MEA and GAU methods are different, and it is interesting to combine them (see the results obtained with the MEA-GAU method). The MEAGAU-UNI method permits to add some points in locally flat regions (see Figure 4).

\subsection{Rigidity Constraints in Pathological Cases}

We have developed a registration algorithm for the thoracic region in the presence of pathologies [11]. The advantage of our approach is that it takes into account the tumors, while preserving continuous smooth deformations. We assume that the tumor is rigid and thus a linear transformation is sufficient to cope with its movements between $\mathrm{CT}$ and PET images. This hypothesis is relevant and in accordance with the clinicians' point of view, since tumors are often compact masses of pathological tissue. The algorithm relies on previously segmented structures (lungs and tumors). Landmarks corresponding to homologous points are defined in both images, and will guide the deformation of the PET image towards the CT image. The deformation at each point is computed using an interpolation procedure based on the landmarks, the specific type of deformation of each landmark (depending on the structure it belongs to), and weighted by a distance function, which guarantees that the transformation is continuous. We have shown that a consistent and robust transformation is obtained [11]. 


\subsection{Registration with Rigidity Con- straints and Breathing Model}

Once the different CT meshes are computed and the closest CT mesh, $M_{C}$, is selected, the PET and the original CT (in our example $M_{N}$ ), are registered as follows:

(1) Select landmarks on the CT mesh $M_{C}$ (with Gaussian or/and mean curvatures);

(2) Estimate corresponding landmarks on the PET (using the Iterative Closest Point algorithm [2]);

(3) $i=C$;

(4) Track landmarks from $M_{i}$ to the next CT mesh $M_{i+1}$;

(5) If $M_{i+1}=M_{N}$, go to step (6) else go to step (4) with $i=i+1$;

(6) Register (with the method summarized in Section 4.2) the PET and the original CT using the estimated correspondences.

In step (1), the four variants presented in Section 4.1 can be used. The breathing model is used in step (4). The landmark points selected on $M_{C}$ are tracked on the meshes estimated with the breathing model. Consequently, we can assume that the corresponding landmarks selected on the original CT are correct (and actually they represent the same anatomical point) and follow the deformations of the lungs during the respiratory cycle.

\section{Results and Discussion}

In this section we present some results we have obtained using the general methodology described in Section 3 and the registration method summarized in Section 4.

We have applied our algorithm on a pathological case exhibiting one tumor and on a normal case. We have one PET and two CT images for each case. First, the breathing model is computed using the meshes of the lungs segmented on CT data. Then, we compare 10 (regularly distributed) instants of the generated model with the lung surface segmented from the PET (meshes have more than 40000 nodes). Figure 5 shows the results of surface comparison between the PET surface, for two instants from the CT data set: the closest and the end-inspiration.

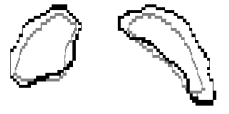

(a) $D_{R M S}=12.1$
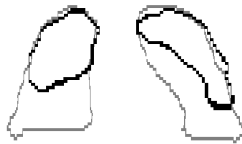

(b) $D_{R M S}=24.2$
Figure 5. Superimposition of the contours of the PET (black) and the CT lungs (grey) at two instants of the breathing cycle: (a) closest $\left(M_{C}\right)$ and (b) end-inspiration $\left(M_{N}\right)$.

The results obtained with the proposed algorithm are physically-based and more realistic than results obtained by registering the PET directly with the original CT. First results confirm this statement as shown in Figures 6 and 7. It can be observed that the result of the registration by a direct method (Figure 6(d)) produces unrealistic deformations in the region between the lungs. With the proposed algorithm (Figure 6(h)), the result is visually more accurate. The RMS distance between the registered PET lungs and the original CT lungs is reduced to $11.8 \mathrm{~mm}$. In Figure 7 the improvement of the results is clearly illustrated for the normal case in the region of the right lung close to the liver. All these results have been obtained by using landmarks determined by the combination of mean and Gaussian curvatures plus a uniform selection. This variant provided visually better results although further validation is necessary. (a)

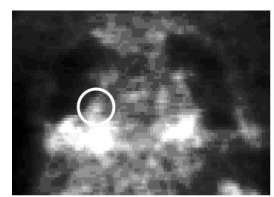

(c)

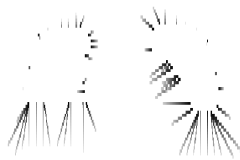

(e)

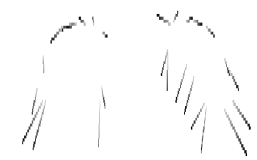

(g)

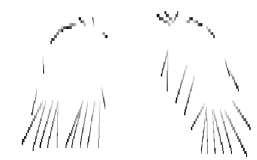

(b)

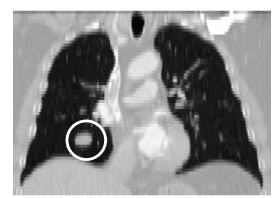

(d)

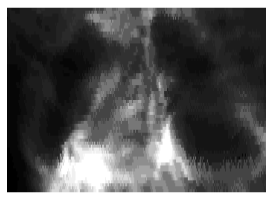

(f)

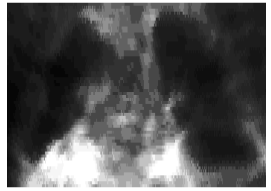

(h)

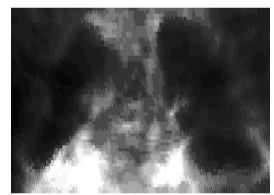

Figure 6. Original PET (a) and CT (b) images with tumor (surrounded by a white circle). Correspondences between the selected points in the PET image and in the CT image are shown in (c), (e) and (g). Registered PET data is shown in (d) for the direct method and in (f) and (h) for the method with the breathing model with the variant combining mean and Gaussian curvatures in (e)-(f) and adding uniformly distributed landmarks in $(\mathrm{g})-(\mathrm{h})$. Illustrations are provided in $2 \mathrm{D}$ for the sake of readability.

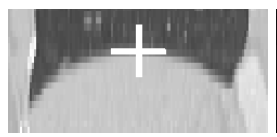

(a)

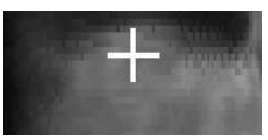

(b)

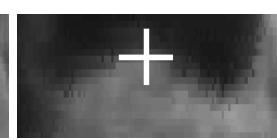

(c)
Figure 7. Details of registration on the bottom part of right lung, in a normal case, (a) CT, (b) PET registered without breathing model, (c) with breathing model. The white crosses correspond to the same coordinates.

\section{Conclusion and Future Work}

We have developed a CT-PET landmark-based registration method that uses a breathing model to guarantee phys- 
iologically plausible deformations. The method consists in computing a deformation guided by a breathing model. We also proposed and studied four variants, based on curvature, to select landmarks. Initial results on a pathological case and a normal case are very promising and show the improvement brought by the breathing model. Our algorithm avoids undesired tumor misregistrations and preserves tumor geometry and intensity. Moreover, as the tumor in CT and PET has not necessarily the same size and shape, the registration of these two modalities is very useful because all the information of the PET image is preserved. This is very important in order to know the true extension of the pathology for diagnosis and for the treatment of the tumor with radiotherapy, for example. Moreover, we highlight the best variant to detect landmarks: to uniformly select landmarks by combining mean and Gaussian curvatures.

We are currently performing a deeper evaluation on a larger database, in collaboration with clinicians. Future work includes a refined "snapshot" selection, using further subdivisions of time intervals, a more precise characterization of the tumor movement and its influence on the breathing, and a comparison of the the proposed method with other approaches which include rigid objects in the registration.

Acknowledgments: This work has been partially funded by an ANR grant: ANR-05-BLANC-0081.

\section{References}

[1] W. Beil, K. Rohr, and H. Stiehl. Investigation of approaches for the localization of anatomical landmarks in 3D medical images. In Computer Assisted Radiology and Surgery (CARS), pages 265-270, Apr. 1997.

[2] P. Besl and N. McKay. A Method for Registration of 3-D Shapes. IEEE TPAMI, 14(2):239-256, 1992.

[3] M. Betke et al. Landmark detection in the chest and registration of lung surfaces with an application to nodule registration. Medical Image Analysis, 7(3):265-281, 2003.

[4] O. Camara et al. Explicit Incorporation of Prior Anatomical Information into a Non-Rigid Registration of Thoracic and Abdominal CT and 18-FDG Whole-Body Emision PET Images. IEEE TMI, 26(2), 2007.

[5] T. Guerrero et al. Elastic image mapping for 4-D dose estimation in thoracic radiotherapy. Radiation Protection Dosimetry, 115(1-4):497-502, 2005.

[6] T. Hartkens et al. Using Points and Surfaces to Improve Voxel-Based Non-Rigid Registration. In MICCAI, volume 2489, pages 565-572, Tokyo, Japan, 2002.

[7] W. Lavely et al. Automatic registration of PET and CT studies for clinical use in thoracic and abdominal conformal radiotherapy. Medical Physics, 31(5):1083-1092, 2004.

[8] J. Maintz and M. Viergever. A Survey of Medical Image Registration. Medical Image Analysis, 2(1):1-36, 1998.

[9] J. McClelland et al. A Continuous 4D Motion Mmodel from Multiple Respiratory Cycles for Use in Lung Radiotherapy. Medical Physics, 33(9):3348-3358, 2006.
[10] J. Mead. Measurement of Inertia of the Lungs at Increased Ambient Pressure. JAP, 2(1):208-212, 1956.

[11] A. Moreno et al. Non-linear Registration Between 3D Images Including Rigid Objects: Application to CT and PET Lung Images With Tumors. In DEFORM06, pages 31-40.

[12] S. Nehmeh et al. Four-dimensional (4D) PET/CT imaging of the thorax. Physics in Medecine and Biology, 31(12):31793186, 2004.

[13] J. Pluim and J. Fitzpatrick. Image Registration. IEEE TMI, 22(11):1341-1343, 2003.

[14] G. Rizzo et al. Automatic registration of PET and CT studies for clinical use in thoracic and abdominal conformal radiotherapy. Physics in Medecine and Biology, 49(3):267-279, 2005.

[15] T. Rohlfing et al. Modeling Liver Motion and Deformation During the Respiratory Cycle Using Intensity-Based FreeForm Registration of Gated MR Images. Medical Physics, 31(3):427-432, 2004.

[16] K. Rohr et al. Landmark-based elastic registration using approximating thin-plate splines. IEEE TMI, 20(6):526-534, 2001.

[17] A. Santhanam. Modeling, Simulation, and Visualization of 3D Lung Dynamics. PhD thesis, University of Central Florida, 2006.

[18] A. Santhanam et al. Modeling Simulation and Visualization of Real-Time 3D Lung Dynamics. IEEE TITB, 2007. In press.

[19] D. Sarrut et al. Non-rigid registration method to assess reproducibility of breath-holding with $\mathrm{ABC}$ in lung cancer. International Journal of Radiation Oncology-Biology-Physis, 61(2):594-607, 2005.

[20] W. Segars et al. Study of the Efficacy of Respiratory Gating in Myocardial SPECT Using the New 4-D NCAT Phantom. IEEE TNS, 49(3):675-679, 2002.

[21] R. Shekhar et al. Automated 3-Dimensional Elastic Registration of Whole-Body PET and CT from Separate or Combined Scanners. The Journal of Nuclear Medicine, 46(9):1488-1496, 2005.

[22] T. Sundaram and J. Gee. Towards a Model of Lung Biomechanics: Pulmonary Kinematics Via Registration of Serial Lung Images. Medical Image Analysis, 9(6):524-537, 2005.

[23] W. Vogel et al. Correction of an image size difference between positron emission tomography (PET) and computed tomography (CT) improves image fusion of dedicated PET and CT. Physics in Medecine and Biology, 27(6):515-519, 2006.

[24] J. B. West et al. Hybrid point-and-intensity-based deformable registration for abdominal ct images. In SPIE Medical Imaging, volume 5747, pages 204-211, 2005.

[25] J. Wolthaus et al. Fusion of respiration-correlated pet and ct scans: correlated lung tumour motion in anatomical and functional scans. Physics in Medecine and Biology, 50(7):1569-1583, 2005.

[26] B. Zitovà and J. Flusser. Image Registration Methods: A Survey. Image and Vision Computing, 21:977-1000, 2003.

[27] V. Zordan et al. Breathe Easy: Model and Control of Human Respiration for Computer Animation. Graphical Models, 68(2):113-132, 2006. 